\title{
Jóvenes y violencia en el ámbito escolar. Un análisis de las representaciones desde la prensa argentina $(1993-2011)^{1}$
}

\section{Youth and Violence in the School Environment. An Analysis of the Representations from the Argentine Press (1993-2011)}

Recibido: 13 de junio de 2016 - Revisado: 23 de agosto de 2016 - Aceptado: 09 de noviembre de 2016.

Virginia Saez ${ }^{2}$

\section{Resumen}

Este artículo presenta los resultados de una investigación que analiza la representación de los jóvenes estudiantes en la mediatización del fenómeno de las violencias en las escuelas de la ciudad de La Plata, Argentina, del período 1993-2011. Dadas las características del objeto de estudio el abordaje metodológico fue cualitativo y la etapa de análisis de la información se realizó en el marco del análisis socioeducativo del discurso. Entre los resultados obtenidos, observamos que en las formas de designar al "alumno violento" se presentan dos dimensiones para caracterizar a los jóvenes: la patológica y la cognitivoacadémica. Dada la originalidad del estudio, aporta un antecedente para futuras indagaciones que aborden la asociación de los jóvenes con la violencia en el espacio escolar.

\section{Palabras clave}

Violencia, medios de comunicación, estudiantes.

\begin{abstract}
This article presents the results of a research that analyzes the representation of young students in the mediatization of the phenomenon of violence in schools in the city of La Plata, Argentina, from 1993-2011. Given the characteristics of the study object, the methodological approach was qualitative and the information analysis stage was carried out within the framework of the socio-educational analysis of the discourse. Among the results obtained, we observe that in the forms of designating the "violent student" two dimensions are presented to characterize the young: the pathological and the cognitiveacademic. Given the originality of the study, it provides a background for future research that addresses the association of young people with violence in the school space.
\end{abstract}

\section{Keywords}

Violence, media, students.

\footnotetext{
${ }^{1}$ Artículo de investigación vinculado al Programa de Investigación sobre Transformaciones Sociales, Subjetividad y Procesos Educativos del Instituto de Investigaciones en Ciencias de la Educación de la Universidad de Buenos Aires.

${ }^{2}$ Doctora en Educación y Magíster en Educación, Pedagogías Críticas y Problemáticas Socioeducativas de la, Universidad de Buenos Aires; becaria posdoctoral del Consejo Nacional de Investigaciones Científicas y Técnicas de la República Argentina. Desarrolla sus investigaciones en el marco del Programa de Investigación sobre Transformaciones Sociales, Subjetividad y Procesos Educativos del Instituto de Investigaciones en Ciencias de la Educación de la Universidad de Buenos Aires.

Correo electrónico: saezvirginia@ hotmail.com

Para citar este artículo use: Saez, V. (2017). Jóvenes y violencia en el ámbito escolar. Un análisis de las representaciones desde la prensa argentina (1993-2011). Civilizar Ciencias Sociales y Humanas, 17(33), 177-188. doi: $10.22518 / 16578953.907$
} 


\section{Introducción}

En Argentina, desde hace poco más de una década, se desarrollan estudios sobre las violencias en la escuela, generados tanto por centros gubernamentales como por instituciones académicas universitarias (Kaplan, 2006 y 2009; Kaplan, Krotsch y Orce, 2012; Míguez, 2008; Observatorio Argentino de Violencia en las Escuelas, 2009 y 2011; Unicef y FLaCSo, 2011; entre otros). A nivel internacional autores como Debarbieux (2008) y Furlán (2005), resaltan la incidencia de la irrupción mediática de la temática en la conformación de este objeto de estudio.

Ahora bien, los medios de comunicación, al escenificar la violencia, derivan en la estigmatización de los jóvenes (Núñez, 2007; Saintout, 2009), al considerarlos como los portadores del conflicto (Cerbino, 2012; Rey, 2005). En este sentido, los análisis efectuados por el Observatorio de Jóvenes, Comunicación y Medios de la Universidad Nacional de La Plata (2012) verifican la presencia de una serie de casos que marcaron la emergencia de los jóvenes en los medios: ya sea como víctimas o victimarios, estos aparecen en la prensa ligados a casos de violencia. Esta homologación mediática entre juventudes y violencias pone de relieve la necesidad de realizar estudios sobre el tratamiento discursivo y las asociaciones de sentido referidas a este grupo etario, para generar procesos reflexivos y prácticas de intervención que permitan confrontar al sentido común hegemónico que lo penaliza.

Dados estos antecedentes, en el presente trabajo se analizarán los discursos mediáticos sobre las violencias en las escuelas y su relación con las representaciones de juventud implícitos. Sostenemos como hipótesis que dicha mediatización tiene efectos simbólicos sobre los procesos de estigmatización de los jóvenes.

\section{Aspectos metodológicos}

La presente investigación caracteriza y analiza, desde una perspectiva socioeducativa, las prácticas discursivas producidas por la prensa escrita de la ciudad de La Plata, Argentina, sobre el fenómeno de las violencias en la escuela, en el período 1993-2011. Desde esta perspectiva se considera que las violencias en la escuela no son un correlato mecánico de la violencia social, aunque es allí donde se originan y cobran su sentido más hondo. Ciertas mediaciones intervienen en la escuela para que esta posibilite algo distinto que los comportamientos brutales que suscitan las sociedades capitalistas salvajes (Kaplan, 2009). El objetivo específico consistió en analizar e interpretar los discursos de los diarios sobre las violencias en las escuelas, en relación con las categorías "alumno violento" y "alumno no violento".

El año de inicio del relevamiento fue seleccionado porque los trabajos precedentes afirman que en 1990 el fenómeno de las violencias en las escuelas comienza a consolidarse como objeto científico. Como se mencionó en el apartado anterior, la presencia de hechos nombrados como "violencia escolar" en los medios de comunicación masiva lleva, en gran medida, a instalar el debate dentro de la opinión pública, en las agendas de los organismos estatales y en los campos académicos y de investigación. Ahora bien, aunque se tomaron artículos de prensa publicados a partir de 1990, se encontró que la primera nota sobre violencia en las escuelas aparece en el año 1993.

Es necesario destacar que se eligen los medios gráficos dado que son una referencia dominante y marcan tendencia informativa para el resto de los medios de comunicación (Bonilla-Vélez \& Tamayo-Gómez, 2007) y porque interesa la materialidad de los diarios y la potencialidad del texto escrito para observar las distintas formas de nominación del fenómeno ${ }^{1}$. Se seleccionaron los medios de la ciudad de La Plata porque es la capital de la provincia de Buenos Aires y es considerada su principal centro político, administrativo y educativo. Estas características la hacen significativa del presente estudio, por ser un espacio social a 
partir del cual se toman decisiones que afectan a todas las provincias de la República Argentina.

El abordaje metodológico es cualitativo, y el diseño de investigación asume un carácter exploratorio. El muestreo es intencional: se seleccionaron las prácticas discursivas que aportaran información de interés en relación al objetivo estipulado. La investigación fue sincrónica y se examinó el objeto de estudio en el período comprendido entre 1993 y 2011. No se pretendió analizar cómo han ido modificándose las representaciones mediáticas a lo largo del lapso elegido, sino observar las continuidades en la caracterización de los jóvenes en episodios de violencia en el espacio escolar, lo que aportó bases connotativas para describir el fenómeno. Se abordaron todas las secciones de cada periódico y en el conjunto de estos medios, se halló gran cantidad de semejanzas y pocas diferencias, por tal motivo no se realizó una distinción analítica por cada diario.

Por otra parte, como se demostró en trabajos anteriores (Saez, 2015) existen tensiones y debates presentes en los estudios sobre medios de comunicación que abordan temáticas sociales: corrientes como la Lingüística Crítica (Raiter \& Zullo, 2008) y la del Análisis Crítico del Discurso (Van Dijk, 2007) evidencian que no hay una forma única de abordar los discursos mediáticos y manifiestan su preocupación por la reproducción de la desigualdad. Ya que se comparte esta inquietud, la presente investigación realizó el tratamiento de los datos en el marco del análisis socioeducativo del discurso (Martín-Criado, 2014) pero no los abordó con el encuadre metodológico lingüístico-discursivo característico de dichas corrientes. Esta metodología es de relevancia para

[...] dilucidar el juego de tensiones y ambivalencias en que se mueven prácticas y discursos (...) y ver las estrategias simbólicas para legitimar o deslegitimar a los distintos sujetos y sus prácticas - de ahí el énfasis en situar todo discurso en un espacio de discursos y todo enunciado en la estrategia general de presentación de sí [...] (Martín Criado, 2014, p.133).
La línea de análisis que se sigue postula una interrelación entre las noticias y la práctica social que las produce, por eso referiremos a práctica discursiva. Desde esta perspectiva el uso de ciertos actos de nombramiento y clasificación en las notas estará determinado por las convenciones socialmente aceptadas para el discurso en el que se inserta. A su vez, los límites de ese discurso estarán relacionados con las condiciones de reproducción/ transformación que posibilitan las estructuras sociales existentes. El desafío consistió en establecer dimensiones de descripción y análisis para explicar el tratamiento de la clasificación "alumno violento".

Respecto a la contextualización de los discursos, es relevante mencionar que las notas analizadas estuvieron atravesadas por una temporalidad educativa particular, en la cual se extendió la escolaridad obligatoria de los jóvenes. En 1993, en la Ley Federal de Educación se extiende la obligatoriedad a octavo y noveno año, y en 2006, en la Ley de Educación Nacional se establece la escuela secundaria obligatoria. Estos aspectos son relevantes en la lucha simbólica por la representación de la escuela y los jóvenes. Uno de los propósitos de esta investigación es aportar una alternativa teórica y práctica frente a aquellas posiciones que estigmatizan a los estudiantes como sujetos peligrosos de los cuales habría que resguardarse.

\section{Las representaciones de los jóvenes estudiantes en los discursos mediáticos}

En este estudio se identifican y analizan los discursos mediáticos sobre las violencias en las escuelas en relación con las categorías "alumno violento" y "alumno no violento". Se indaga sobre cómo los comportamientos tipificados como violentos por las notas periodísticas son relacionados con otros atributos (personales y sociales) a partir de los cuales se construye una representación diferenciada entre "jóvenes violentos" y otra de "jóvenes no violentos". 
Partiendo del abordaje del análisis del discurso socioeducativo, se identifican actos de nombramiento, clasificación y modos de designar que permiten, bajo su evidencia, observar algunas formas de visibilizar a los jóvenes en el espacio escolar a través de la noticia. Se observa un "modo de reconocimiento adulto" sobre los jóvenes, el cual no se interesa por resaltar aspectos creativos o por destacar los aportes reales de este grupo etario dentro de las dinámicas sociales que conforman los relatos cotidianos de los periódicos analizados. Los significados que circulan trazan perfiles estereotipados que obstaculizan la edificación de un reconocimiento.

Se ha trabajado con las formas de designar, nominar, adjetivar al "alumno violento" y al "alumno no violento". En todos los casos se interpretó a partir de las categorías formuladas por los artículos de prensa. Desde la perspectiva de esta investigación, estos términos son adjetivaciones o atribuciones con un contenido expreso y uno implícito, es decir, con una matriz de significación oculta (Kaplan, 2008). En este sentido, las formas de designar al "alumno violento" y al "alumno no violento" aluden al repertorio con el que el discurso periodístico categoriza a los estudiantes.

Una primera lectura de los artículos de prensa, nos permite encontrar adjetivos que pertenencen a dos dimensiones, a saber: dimensión patológica y dimensión cognitivo-académica. En la primera se reúnen los modos de designar y las atribuciones brindadas por las notas periodísticas que sugieren caracterizaciones psicológicas y patológicas; en la segunda se reúnen los modos de designar y las atribuciones brindadas por las notas periodísticas que aluden al ámbito cognitivo-académico.

\section{La dimensión patológica}

Las representaciones de los discursos mediáticos del par "alumno violento-alumno no violento" aluden a caracterizaciones psicológicas y patológicas para definir cada polo del par. A continuación se citan algunos ejemplos:
"Caso alumno golpeador: pedirán nueva pericia psiquiátrica" (Hoy, 25 de agosto de 1996, p. 08).

"Locura adolescente. La jueza Lasca consideró que el chico 'está tratando de llamar la atención, porque tiene algunos problemas'. Luego de haber contado su versión en el juzgado, volvió a su casa con su padres, que 'están comprometidos con la situación de su hijo, que quedó bajo tratamiento psiquiátrico', indicó la magistrada" (Hoy, 07 de junio de 2005, p. 06).

Cabe destacar que en los artículos encontrados son recurrentes ciertas nominaciones (tímidos, tranquilos, callados, de conductas extrañas), que dan cuenta del sentido y del peso que las coberturas periodísticas otorgan a la determinación biológica y patológica sobre el comportamiento de los estudiantes. Se va describiendo un perfil psicológico para los "jóvenes violentos-alumnos violentos": Observemos:

“"Es un pibe tranquilo, de perfil bajo y callado. Nunca hubo un problema con él, siempre tuvo una excelente integración y buen comportamiento social', declaró un profesor que hasta ayer lo tenía a su cargo. El chico que desencadenó la tragedia fue descrito por sus propios compañeros como 'tímido', 'callado', de 'conductas extrañas, pero no violentas' y con escasa integración al resto de los alumnos, aunque casi siempre formaba parte del equipo de fútbol de la división, ya que todos los sábados atajaba en un equipo de la zona" (Sin Autor, Hoy, 29 de septiembre de 2004, p. 07).

Las caracterizaciones psicológicas y patológicas aparecen con mayor frecuencia en los modos de designar al "alumno violento". En la producción de las nominaciones se recurre a adjetivaciones más brutales y directas. Kaplan (2008) señala "es el lado negativo del par el que expresa el sentido más profundo, y más inconsciente, desde un punto de vista sociológico" (p. 147).

De este modo, se le adjudica la negatividad a la individualidad del alumno. Desde el discurso autorizado, los jóvenes son estudiados, entre otros, por profesionales especializados como 
psicólogos o psiquiatras, quienes determinan narrativas anómalas que configura nuevas forma de actuación. Como sostiene Bourdieu (2014), el poder de las palabras es el poder delegado del portavoz, y su enunciado es un testimonio de la garantía de la delegación de la que ese sujeto está investido "...El uso del lenguaje, que implica tanto la manera como la materia de discurso, depende de la posición social del locutor, posición que rige el acceso que este pueda tener con la lengua institucional, la palabra oficial, ortodoxa, legítima" (pp. 88-89).

Otra característica de esta dimensión es que, para describir al "alumno violento", los artículos de prensa vinculan a los jóvenes con adicciones. Veamos un ejemplo:

"Droga más alcohol en los jóvenes, combinación que genera violencia" (Sin Autor, El Día, 18 de agosto de 1993, p. 12).

Asimismo, las adicciones son articuladas con problemáticas morales. A continuación presentamos algunos enunciados que revelan esta mirada:

"De su conducta moral podríamos decir que es tan deficiente como su instrucción. Del sentido religioso diremos que es casi una especie en extinción. Del cariño y respeto a los padres hay bastantes quejas. La amistad se basa en hechos fortuitos y aleatorios. Por lo tanto, una estructura semejante es fácil presa del flagelo de los narcóticos y alucinógenos, pandemia de nuestro tiempo. Por supuesto que no toda la juventud está mal encaminada, pero hay un grupo muy importante que actúa como la manzana podrida en el recipiente de las sanas: contagia. ¿Qué debemos hacer?" (Sin Autor, El Día, 17 de abril de 1993, p. 13).

$\mathrm{Y}$ en algunos casos, con trastornos psicosomáticos, perversiones y transgresiones. Observemos:

"Las patologías actuales responden a estructuras narcisistas, incluyendo las tóxicas como las adicciones, los trastornos psicosomáticos, las perversiones y las transgresiones. Desde ya, todas las manifestaciones no psicóticas, que son otras", explica la psicóloga Patricia Maestri, especialista en adolescencia. Transgresor es el que roba, el violento, etc. (Sin $\mathrm{Au}-$ tor, El Día, 20 de marzo de 1994, p. 14).

Dadas las afirmaciones presentadas, estamos en condiciones de plantear que las adhesiones de las coberturas periodísticas a la dimensión patológica son el factor determinante de comportamiento del "alumno violento". Estos modos de designar van estableciendo construcciones de sentido que vinculan un perfil psicológico para los "jóvenes violentos" - "alumnos violentos" (tímidos, tranquilos callados, de conductas extrañas) con adicciones, problemáticas morales, trastornos psicosomáticos, perversiones y transgresiones. El individuo aparece como causa de su propio comportamiento. Sin embargo, como sostiene Bourdieu (2012), la conducta requiere de esquemas aprendidos socialmente:

El conocimiento práctico del mundo social que supone la conducta "razonable" en ese mundo elabora unos esquemas clasificadores (o si se prefiere, unas "formas de clasificación", unas "estructuras mentales", unas "formas simbólicas", expresiones todas ellas que, si se ignoran connotaciones, son más o menos intercambiables), esquemas históricos de percepción y apreciación que son producto de la división objetiva de clases (clases de edad, clases sexuales, clases sociales) y que funcionan al margen de la conciencia y del discurso (p. 479).

Ahora bien, en algunos reportajes del diario Hoy se incluye la voz de los jóvenes, quienes se muestran resistentes a estas reiteradas asociaciones con las adicciones. Veamos el siguiente extracto:

\footnotetext{
“'La juventud no es sinónimo de droga', dijo un estudiante de una escuela técnica de la ciudad. 'No es como el acné juvenil. No es algo privado de la edad. Ni siguiera es una característica de ese período', señaló un docente secundario que prefirió no darse a conocer" (Sin Autor, Hoy, 27 de octubre de 1994, p. 12).
} 
En la lucha por la producción y la imposición de la visión legítima del mundo social no se obtiene nunca el monopolio absoluto. Como sostiene Bourdieu (2009): "En realidad, hay siempre, en una sociedad, conflicto entre los poderes simbólicos que tienden a imponer la visión de las divisiones legítimas, es decir a construir grupos" (p. 140). A pesar de tener voz en uno de los medios, el juego beneficia a los profesionales del verbo, de la palabra autorizada, en tanto aparecen con mayor recurrencia y en los cuatro medios.

En síntesis, estos modos de designar legitiman cierta mirada sobre los estudiantes y la escuela, y tienden a construir las experiencias de violencia en la escuela como fenómenos del orden de lo individual. A través de estas prácticas discursivas se da por supuesta cierta idea o modelo de humanidad y se retoma la idea normativa, evidente, y supuestamente universal de hombre. Desde el punto de vista de las prácticas discursivas de la prensa gráfica de La Plata, el concebir la violencia como una característica intrínseca o esencial del joven sustituye las condiciones sociales y escolares de producción del vínculo, que difiere según los contextos institucionales y los grupos sociales por los atributos naturales del individuo.

A partir de una perspectiva crítica (Kaplan, 2006, 2009 y 2013) se analiza cómo irrumpe la violencia en contextos sociales (valores, instituciones) cuyas interrelaciones incluyen que su proyecto de vida, para gran parte de los sujetos individuales y colectivos, carezca de sentido. Norbert Elias es uno de los sociólogos que más ha intentado penetrar en la sociedad de los individuos, en el difícil vínculo entre la existencia social y la existencia individual, en cómo los sujetos dan sentido y se representan sus vidas y la de los otros en interacción. Así, la violencia es siempre relacional y necesita ser interpretada bajo esa cualidad.

En todo caso, más allá de ciertas patologías que podrían afirmar conductas violentas personales, lo cierto es que, "aun en esos casos individuales" es preciso ampliar la base interpretativa de la acción y situarla en los modos de socialización que cada sociedad privilegia y legitima. "El riesgo, si no, es tratar a los 'casos sociales' como 'casos patológicos"” (Kaplan, 2009, p. 23).

Se considera de gran valor la perspectiva histórica de Norbert Elias para la interpretación de los actos de violencia en nuestras sociedades, dado que estos muchas veces son explicados mediante la asociación a ciertos individuos o grupos sociales. Las ideas de este autor convocan a los investigadores a la "vigilancia epistemológica" respecto de la insistencia en la atribución de esos actos a designios personales.

\section{La dimensión cognitivo-académica}

Las representaciones de los discursos mediáticos del par "alumno violento-alumno no violento" aluden a caracterizaciones del plano intelectual y del ámbito cognitivo-académico, como el rendimiento escolar, las calificaciones y la racionalidad de sus actos para definir cada polo del par. En las prácticas discursivas de la prensa platense aparece una asociación entre el par "alumno violento-alumno no violento" con el rendimiento escolar, a través de la caracterización del "alumno violento" como uno de "bajas calificaciones". Veamos una nota:

“'Rafael Junior tenía bajas calificaciones', señaló uno de los docentes que hasta ayer lo tenía a su cargo" (Sin Autor, Hoy, 29 de septiembre de 2004, p. 07).

Hay un vínculo entre las evaluaciones académicas y la tipificación del par "alumno violento-alumno no violento" de las prácticas discursivas de la prensa platense. Se trasladan los procesos sociales de distinción presentes en el acto de evaluación del sistema educativo para diferenciar los polos de la clasificación descrita.

Asimismo, al "alumno violento" se lo caracteriza como "poco aplicado", en referencia al oficio de alumno implícito en la vida en 
las aulas, lo que Perrenoud (2008) plantea como "currículum moral o de los objetivos no cognitivos (orden, limpieza, servicio)". Kaplan (2008) menciona este aspecto como un elemento de evaluación que se lo identifica con la inteligencia. Observemos el siguiente caso:

"Rafael S. ocupaba uno de los últimos lugares del aula, de espaldas a la pared, sitio por lo general reservado para alumnos poco interesados en el estudio. Aunque contrariamente a la histórica lógica juvenil, Junior era uno de los más aplicados del curso" (Sin Autor, Hoy, 29 de septiembre de 2004, p. 07).

Otra relación que emerge de las notas periodísticas es la designación de los actos del "alumno violento" como "irracionales", se alude a la falta de discernimiento moral e incapacidad para distinguir entre aquello que está bien y aquello que no: sus manifestaciones violentas son producto de "un acto irracional" (Sin Autor, Hoy, 7 de septiembre de 2005, p. 08). Ya desde el comienzo del período se registran "representaciones groseras de la alteridad (...) desprovistas de sutileza, ligadas a una lógica de la diferenciación" (Wieviorka, 2009, p. 158). Veamos el siguiente enunciado:

"Cada vez más jóvenes se convierten en monstruos" (Sin Autor, Hoy, 25 de agosto de 1996, p. 06).

Estos calificativos marcan una preocupación por controlar los jóvenes, "reducirlos a una fuerza social prácticamente animal, en todo caso muy inferiorizada, cuyos riesgos de revuelta o de cólera y de rabia exigen una gran vigilancia" (Wieviorka, 2009, p. 160). Se hace una atribución de sentido que asocia al "alumno violento" con la irracionalidad de una barbarie que ataca al espacio escolar a través de actos de vandalismo y de violencia hacia los actores de la comunidad educativa. A continuación citamos algunos enunciados que evidencian esta mirada:

"Los escolares británicos volvieron a ser blanco de la irracionalidad de jóvenes desmedidos." (Sin Autor, Hoy, 9 de julio de 1996, p. 07).
"De un momento a otro la paz educativa se alteró radicalmente: cientos de chicos corrían de un lado al otro del patio para impedir que los encapuchados les pegaran un tomatazo o un huevazo. Docentes y preceptores inútilmente intentaban mantener la calma y protegerse frente a la irracional agresión" (Sin Autor, Hoy, 7 de agosto de 1999, p. 08).

Dada esta caracterización directa y brutal asociada a la barbarie se señala que el "alumno violento" ha de ser objeto de intervención en la escuela; la propuesta es, durante los años escolares, civilizar y disciplinar a estos jóvenes mediante la vigilancia y el encauzamiento de su conducta. Algunas citas que muestran este argumento:

\footnotetext{
"La escuela es la que debe inculcar en estos jóvenes las buenas costumbres" (Sin Autor, El Día, 6 de abril de 1993, p. 13).

"La educación de la juventud, una tarea que debe mancomunar la escuela para una sociedad civilizada" (Sin Autor, El Día, 17 de abril de 1993, p. 11).
}

En las notas periodísticas se apela al espacio escolar como un lugar de transformación de la juventud bárbara en juventud civilizada, inculcando las buenas costumbres, canalizando las ansiedades generacionales e instalando en el orden cultural por su conexión con la cultura letrada. El "alumno violento" queda asociado a la barbarie primitiva que se mantiene en el orden de la naturaleza. Estas miradas sobre los estudiantes tienen raigambres históricas y reflejan las luchas en las relaciones intergeneracionales (Bourdieu, 2012). Ya las posiciones pedagógicas de Sarmiento $^{2}$ de principios del siglo XX suponen la implantación de un sistema de instrucción pública pensado como dispositivo para alterar la continuidad de las generaciones, como un medio para cambiar su moralidad y sus hábitos (Carli, 2012; Puiggrós, 2006).

A su vez, tiene un correlato con los aportes de la sociología figuracional de Norbert Elias (2009), quien define la violencia y la ci- 
vilización como formas específicas de interdependencias en tanto procesos complementarios. En su estudio sobre la cultura occidental del siglo XVI y la Edad Media evidencia cómo la civilización dependerá del nivel de control de la violencia, del monopolio de los impuestos que permiten constituir una fuerza suficientemente efectiva para imponer la pacificación interna, entre otros aspectos. En la figuración francesa del siglo XVII, Elias analiza la civilización como producto del desarrollo y con intención de expandirse a otros pueblos que se le contraponían por su opuesto: la barbarie. La civilización es pensada no como un estado fijo sino como un proceso donde participan la educación y el refinamiento de las costumbres, para diferenciarse de lo bárbaro y lo no civilizado.

También se encontró en los artículos de prensa la "importación" de términos desde la perspectiva judicial o policial, como la de "menor" para nombrar a los jóvenes que protagonizan las noticias del fenómeno. Desde el ámbito del que proviene este término, refiere a la persona que no alcanzó la mayoría de edad, y designa especialmente a aquellos acusados de cometer un delito. Por tal motivo, su uso en el discurso social conlleva un significado peyorativo, que termina reforzando prejuicios, estigmas y desigualdades sociales. Este nombramiento opera diferenciando entre dos supuestos modelos de infancia y juventud: los niños o jóvenes y los menores. Asimismo, las intromisiones de la terminología judicial están ligadas a las soluciones propuestas en las noticias sobre las violencias en el espacio escolar, aspecto que se desarrolló en trabajos anteriores (Saez, 2015).

A partir de su estatus jurídico, la aplicación de este término a los jóvenes refiere a dos sentidos: menores de edad y menores de razón frente al adulto. Desde los orígenes del sistema escolar argentino hay una asociación entre la identidad jurídica y la identidad educativa. En el discurso sarmientino esta asociación permitió construir la autoridad del maestro, establecer una frontera de edad asentada en lo que establecía el Código Civil para sostener el poder sobre los estudiantes, sin derechos propios y subordinados a la autoridad de los adultos. La minoría de razón de los jóvenes los convierte en seres incompletos, por estar en situación de crecimiento o de exceso de pasión (Carli, 2012).

A través del análisis de las dimensiones presentadas se produce una operación mediática que consiste en yuxtaponer la información de un grupo determinado, los jóvenes, con el problema social de las violencias en el espacio escolar. La prensa platense reproduce y transmite las violencias en el espacio escolar perpetuando estereotipos y prejuicios. Las formas de clasificación, apreciación y evaluación presentadas en los nombramientos contribuyen sistemáticamente a ofrecer una imagen sesgada. Observemos en el siguiente gráfico cómo se construyó la tipificación del "alumno violento" a partir de adjetivos, nominaciones y formas de designar asociadas a cada categoría:

\section{Gráfico 1: Tipificación del alumno violento}

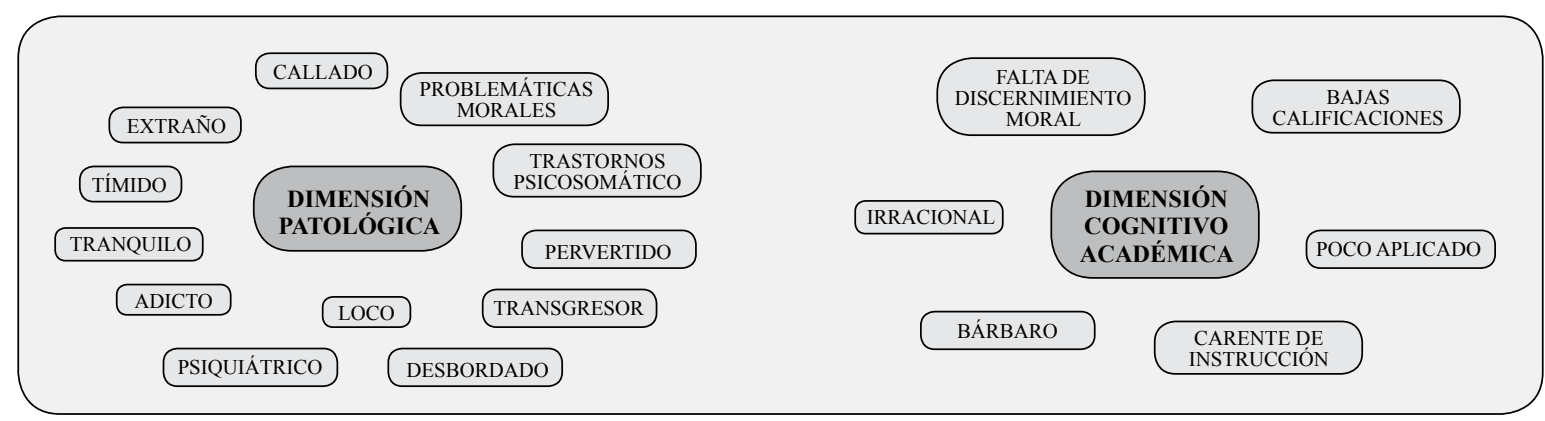

Fuente: Elaboración propia. 
La mediatización del par "alumno violento-alumno no violento" se presenta de forma permanente en el período analizado, asociándose a la imagen de juventud bárbara -juventud civilizada.

\section{Conclusiones}

Desde una perspectiva socioeducativa se han caracterizado las tipificaciones sobre la violencia producidas por la prensa escrita de la ciudad de La Plata sobre el fenómeno de las violencias en las escuelas, en el período de 19932011. Se analizaron los artículos de prensa en relación con las categorías "alumno violento" y "alumno no violento" y se describió cómo los comportamientos representados como violentos son relacionados con otras particularidades (personales y sociales) a partir de los cuales se construye una representación diferenciada entre jóvenes violentos y jóvenes no violentos.

Siguiendo a Elias (2016), "La mayoría de los hombres de todas las sociedades tiene a su disposición toda una gama de expresiones para estigmatizar a otros grupos" (p. 96). Las luchas simbólicas por los actos de clasificación social y el posicionamiento dentro del espacio socialmente jerarquizado pueden observarse en la presentación de la juventud como riesgo. Sin hacer visibles sus derechos, se la considera como problema que se corrige y no como potencialidad que se promueve.

La primera parte del estudio adelantó un análisis de las formas de designar, nominar y adjetivar al par "alumno violento-alumno no violento", desde su contenido expreso e implícito (Kaplan, 2008), para dar paso a la reconstrucción dos dimensiones: la patológica y la cognitivo-académica. Esta categorización constituye un aspecto novedoso en las investigaciones que se centran en la forma en que los medios de comunicación presentan las violencias en el espacio escolar.

Desde la primera dimensión se analizó cómo las etapas de la infancia y la juventud son presentadas en muchos casos desde una mirada que implica una patología de lo social. El descontrol se asocia a otros comportamientos como el consumo en exceso de drogas y alcohol. Aquí nos interrogamos sobre el manejo de los conceptos "inclusión-exclusión", y "ellos-nosotros" presentes en las notas. La representación que los artículos construyen se realiza sobre procesos de diferenciación-identificación centrados en pares dicotómicos jerárquicos: normal-anormal y civilizado-bárbaro, rico-pobre. Las nominaciones $\mathrm{y}$ adjetivaciones socialmente peyorativas con las cuales se mencionan y/o describen a quienes consideran "violentos" operan como actos de clasificación social que los ubican en posiciones inferiores del espacio social y simbólico. Las clasificaciones sociales operan sobre todo a través de oposiciones dualistas y organizan la percepción del mundo social (Bourdieu, 2012).

Vimos cómo se significan los atributos: a los jóvenes que tipifican como "violentos" se les asocian otras prácticas y hábitos como el rendimiento escolar. Estas forman parte de la operación esencializadora de los discursos racistas. Sin embargo, las operaciones de clasificación social son el resultado de una lucha simbólica y no se realizan con base en sustancias sino en relación y comparación a otras propiedades (Bourdieu, 2014). En las formas de designación, con las que se pretende reducir a algo homogéneo fenómenos extremadamente complejos, quedan ubicados en primer plano aquellos aspectos de las violencias que se asocian con las explicaciones realizadas en términos de "anomalías" individuales, de "desviaciones" de la norma, "deficiencias" y "carencias".

El presente estudio permitió un avance en la conceptualización de las violencias en los espacios escolares a través de los medios gráficos de comunicación. Tras el análisis de los discursos se infiere que se construye una representación de los jóvenes estudiantes como sujetos peligrosos desde las dos dimensiones definidas (la patológica y la cognitivo-académica). Constituye un aporte especifico al análisis de los 
modos en que la prensa escrita nos sitúa frente a datos y episodios que refuerzan una serie de creencias sociales que configuran un sentido práctico de la doxa punitiva (Kaplan, 2006). En coincidencia con investigaciones anteriores (Cerbino, 2012; Núñez, 2007; Rey, 2005; Saintout, 2009), las formas en que los medios de comunicación presentan la violencia y la juventud están vinculadas con la desigualdad social. Es deseable que los medios participen en el desarrollo de nuevas sensibilidades y disposiciones hacia los jóvenes como sujetos de derecho, contribuyendo a renovar el compromiso social con una sociedad más democrática y más justa.

A modo de cierre es importante destacar la relevancia de esta investigación en tanto amplía la base empírica con la que pensar y discutir cómo a través de los sentidos implícitos en las coberturas se construyen ciertos sujetos sociales como amenazantes. En tal sentido, es el primer estudio en Argentina que aborda las representaciones mediáticas de los estudiantes vinculados con la violencia en el espacio escolar desde una perspectiva socioeducativa.

\section{Notas}

1 Resulta pertinente aclarar que no se examinaron las fotografías presentes en las coberturas, esto se realizará en futuros trabajos.

2 Domingo Faustino Sarmiento fue un político, escritor, docente, periodista, militar y estadista argentino que se desempeñó como gobernador y presidente de su país. Resulta notable que muchos aspectos de la organización del sistema educativo ideado por Sarmiento permanezcan con cambios leves hasta la actualidad (Carli, 2012).

\section{Referencias}

Bonilla-Vélez, J. I., \& Tamayo-Gómez, C. A. (2007). Las violencias en los medios, los medios en las violencias. Revisión y análisis critico de los estudios sobre medios de comunicación y violencia en América Latina 1998-2005. Bogotá: CINEP, Fundación Centro de Investigación y Educación Popular.

Bourdieu, P. (2009). Espacio social y poder simbólico. En, Cosas dichas (pp. 127142). Buenos Aires: Gedisa.

Bourdieu, P. (2012). La distinción. Criterio y bases sociales del gusto. Madrid: Taurus Humanidades.

Bourdieu, P. (2014). ¿Qué significa hablar? Economía de los intercambios lingüisticos. Buenos Aires: Akal.

Carli, S. (2012). Niñez, pedagogía y política. Transformaciones de los discursos acerca de la infancia en la historia de la educación argentina 1880-1955. Buenos Aires: Miño y Dávila.

Caso alumno golpeador: pedirán nueva pericia psiquiátrica. (25 de agosto de 1996). Hoy, p. 06.

Cerbino, M. (2012). El lugar de la violencia. Perspectivas criticas sobre pandillerismo juvenil. Quito: Taurus.

Debarbieux, E. (2008). Les dix commandements contre la violence à l'école. État des lieux. París: Editions Odile Jacob.

Detuvieron a cuatro niños por robar en una escuela. (9 de julio de 1996). Hoy, p. 07.

Droga más alcohol en los jóvenes, combinación que genera violencia. (18 de agosto de 1993). El Día, p. 12.

Elias, N. (2009). El proceso de la civilización. México: Fondo de Cultura Económica.

Elias, N. (2016). Ensayo teórico sobre las relaciones entre establecidos y marginados. 
En N. Elias \& J. L. Scotson (Eds.), Establecios y Marginados. Una investigación sociológica sobre los problemas comunitarios (pp. 73-186). México: Fondo de Cultura Económica.

Furlán, A. (2005). Problemas de indisciplina y violencia en la escuela. Revista Mexicana de Investigación Educativa, 10(26), 631639.

¿Hay consumo de droga en las escuelas? (27 de ocubre de 1994). Hoy, p. 12.

Infierno en el aula. (29 de septiembre de 2004). Hoy, p. 07.

Kaplan, C. V. (Dir.) (2006). Violencias en plural. Sociología de las violencias en la escuela. Buenos Aires: Miño y Dávila.

Kaplan, C. V. (Coord.) (2008). Comportamiento individual y estructura social: cambios y relaciones. Una lectura desde Norbert Elias. En, La civilización en cuestión. Escritos inspirados en la obra de Norbert Elias (pp. 151-168). Buenos Aires: Miño y Dávila.

Kaplan, C. V. (Dir.) (2009). Violencia escolar bajo sospecha. Buenos Aires: Miño y Dávila.

Kaplan, C. V. (2013): Culturas estudiantiles. Sociología de los vínculos en la escuela. Buenos Aires: Miño y Dávila.

Kaplan, C. V., Krotsch, L., \& Orce, V. (2012). Con ojos de joven. Relaciones entre desigualdad, violencia y condición juvenil. Buenos Aires: Editorial de la Facultad de Filosofía y Letras, UBA.

La educación de la juventud, una tarea en la que deben mancomunarse familia y escuela. (17 de abril de 1993). El Día, p. 11.

Los jóvenes platenses. (20 de marzo de 1994). El Día, p. 14.
Los maestros intentan detener la violencia. (7 de agosto de 1999). Hoy, p. 08.

Martín-Criado, E. (2014). Mentiras, inconsistencias y ambivalencias. Teoría de la acción y análisis de discurso. Revista Internacional de Sociología, 72(1), 115-138.

Miguez, D. (2008). Violencias y conflictos en las escuelas: Aproximaciones a una problemática actual. Buenos Aires: Paidos.

Núñez, V. (marzo, 2007). Apuntes acerca de la violencia en niños y jóvenes: una lectura desde la Pedagogía Social. Seminario presencial en el marco del ciclo Aportes para abordar el malestar en la cultura educativa, organizado por el "Programa de psicoanálisis y prácticas socio educativas. FLaCSo. Área de educación”. Argentina.

Observatorio Argentino de Violencia en las Escuelas (2009). Violencia en las escuelas. Investigaciones, resultados y políticas de abordaje en prevención. Simposio Francia - Argentina. Recuperado de http://ceapi. files.wordpress.com/2012/04/violencia2. pdf

Observatorio Argentino de Violencia en las Escuelas (2011). Cátedra Abierta. Aportes para pensar las violencias en las escuelas. Recuperado de www.me.gov.ar/ construccion/pdf_observatorio/catedra.pdf

Observatorio de Jóvenes, Comunicación y Medios de la Universidad Nacional de La Plata. (2012). Informe Anual 2012. La Plata: Ediciones EPC de Periodismo y Comunicaicón Social.

Otra vez 700 chicos sin clases por vándalos. (7 de junio de 2005). Hoy, p. 06.

Pedirán nueva pericia psiquiátrica. (25 de agosto de 1996). Hoy, p. 08. 
Perrenoud, P. (2008). La construcción del éxito $y$ del fracaso escolar. Madrid: Morata.

Puiggrós, A. (2006). Sujetos, disciplina y currículum en los orígenes del sistema educativo argentino. Buenos Aires: Galerna.

Raiter, A., \& Zullo, J. (Comps.) (2008). La caja de Pandora. La representación del mundo de los medios. Buenos Aires: La Crujía y Facultad de Filosofía y Letras, UBA.

República Argentina. Ley 24.195 de 1993. Ley Federal de Educación. Boletín Oficial del 5 de mayo de 1993. Senado y Cámara de Diputados de la Nación de Argentina, abril de 1993.

República Argentina. Ley 26.206 de 2006. Ley de Educación Nacional. Boletín Oficial No. 31.062. Senado y Cámara de Diputados de la Nación de Argentina, diciembre de 2006.

Rey, G. (2005). El cuerpo del delito. Representación y narrativas mediáticas de la seguridad ciudadana. Colombia: Centro de competencia en comunicación para América Latina.

Saez V. (2015) Una mirada a la investigación sobre medios, violencia y escuela. Revista Entramado, 11(1), 136-155. doi: 10.18041/entramado.2015v11n1.21117
Saintout, F. (2009). ¿Culturas violentas? La producción mediática de violencias legítimas/ilegitimas y de sujetos viables/ inviables. El caso de las juventudes. Recuperado de http://www.perio.unlp. edu.ar/observatoriodejovenes/node/107

Un nuevo episodio de robo en perjuicio de una escuela. (17 de abril de 1993). El Día, p. 13.

Unicef., \& FLaCSo. (2011). Clima, conflicto $y$ violencia en las escuelas. Argentina: FLaCSo. Recuperado de www.unicef. org/argentina/spanish/clima_conflicto violencia_escuelas.pdf

Van Dijk, T. (2007): Discurso racista (Prólogo). En J. J. Igartua \& Carlos Muñiz (Eds.), Medios de comunicación y sociedad. Salamanca: Ediciones Universidad de Salamanca. Recuperado de www.discursos. org/oldarticles/Discurso\%20racista.pdf.

Vandalismos que no se previenen ni reprimen con el debido rigor. (6 de abril de 1993). El Día, p. 13.

Vendetta: alumnos del Nacional atacaron el liceo (7 de septiembre de 2005). Hoy, p. 08

Wieviorka, M. (2009). Racismo. Una introducción. Barcelona: Gedisa. 